\title{
The theory of the strain broadened line shapes of spin resonance and optical zero phonon lines
}

\author{
A. M. STONEHAM \\ Theoretical Physics Division, Atomic Energy Research Establishment, Harwell, \\ Berks.
}

MS. received 29th June 1966

\begin{abstract}
A number of spin resonance and zero phonon optical absorption lines are broadened by microscopic strains in the lattice containing the centres observed. In $\mathrm{MgO}$, for example, the electron spin resonance line of $\mathrm{Fe}^{2+}$, the ENDOR line of $\mathrm{Co}^{2+}$ and the zero phonon lines of colour centres all show strain broadening. The strains appear to be due to dislocations and other crystal imperfections. It is shown that these line shapes can be calculated in terms of the statistical distribution of the imperfections and their individual strain fields. The approach can be generalized to cases where other broadening mechanisms are important, such as spin-spin interactions. The method may also be used to obtain information about the statistical distribution of the strain sources from the observed line shape. To illustrate the method the line shape of $\mathrm{MgO}: \mathrm{Fe}^{2+}$ is calculated on the simple, if rather unrealistic, assumption of an isotropic homogeneous distribution of dislocations. The results are in fair agreement with experiment.
\end{abstract}

\section{Introduction}

In both spin resonance and optical spectra lines occur which are broadened by microscopic strains in the lattice containing the centre. The strains themselves are caused by the presence of dislocations and other crystal imperfections. It will be shown in this paper that in such cases the line shape can be calculated in terms of the properties of the individual crystal imperfections responsible for the strains and the statistical distribution of these strain sources.

Zero-phonon optical absorption lines of colour centres have been observed in a number of crystals, such as MgO (Ludlow and Runciman 1965) and LiF (Hughes and Runciman 1965). At low temperatures these lines appear to be broadened solely by the microscopic strains.

Electron spin resonance and ENDOR lines can also be strain broadened, although the interactions between the magnetic ions in the crystal may be important. The most conspicuous symptoms of strain broadening are that the linewidth is larger than expected from spin-spin interactions and independent of the spin concentration, and that, in the electron spin resonance of ions with a non-zero nuclear spin, the linewidth is different for the different hyperfine lines. In several cases this broadening mechanism has been identified convincingly by assuming a particular distribution in magnitude for the microscopic strain components and showing that a variety of features of the observed line shape can be obtained consistently from this distribution. Thus Feher (1964) compared the linewidths of $\mathrm{MgO}: \mathrm{Fe}^{3+}$ and $\mathrm{MgO}: \mathrm{Mn}^{2+}$ and their variation with magnetic field orientation. McMahon (1964) was able to predict the line shapes of the $\Delta M=1$, $\Delta M=2$ and the double-quantum transition lines for $\mathrm{MgO}: \mathrm{Fe}^{2+}$. The present author has shown (Stoneham 1964, Ph.D. Thesis, University of Bristol) that for $\mathrm{MgO}: \mathrm{Co}^{2+}$ 
the ENDOR linewidth and the variation of the electron spin resonance linewidth with hyperfine line and with spin concentration are consistent with broadening by microscopic strains.

In this article the distribution in magnitude of the microscopic strains will be calculated. The observed line shapes can be calculated from this distribution in a straightforward way. The method to be used is the so-called 'statistical method' of Markoff (Markoff 1912, $\S \S 16$ and 33, reviewed by Chandrasekhar 1942). This method was first applied to line shape problems by Margenau (1935) and has been used more recently by Anderson (1951) and by Grant and Strandberg (1964). The method is outlined in $\$ 2$ where the distribution in magnitude of the local strain is found as a function of the strain fields of the individual sources of strain and of the statistical distribution of these sources in the crystal. A number of general features of the result are discussed in $\S 3$, in particular the moments of the distribution and the case of broadening by both spin-spin interactions and strains simultaneously. To illustrate the main features of the method the line shape of the $\Delta M=1$ transition for $\mathrm{MgO}: \mathrm{Fe}^{2+}$ is calculated in $\$ \S 4$ and 5, assuming that this is broadened by a statistically isotropic and homogeneous distribution of dislocations. Such a distribution has the advantage of simplicity rather than realism, for it neglects the subgrain structure of the dislocation distribution in $\mathrm{MgO}$ crystals as grown (Lang and Miuscov 1964). In the circumstances the agreement with experiment is quite good. The theory will be applied to a more realistic distribution of dislocations in a later publication.

\section{The calculation of the strain distribution}

We consider a transition which has energy $\hbar \omega_{0}$ in an unstrained crystal. The trans1tion energy of one of the centres responsible for this line is altered by the local strain to

$$
\hbar \omega=\hbar \omega_{0}+\hbar \omega_{1}\left(\sum_{\imath \jmath} a_{\imath \jmath} e_{\imath \jmath}\right)+\hbar \omega_{2}\left(\sum_{\imath \jmath k l} b_{\imath \jmath k l} e_{\imath \jmath} e_{k l}\right)+\ldots
$$

where $e_{\imath}$ is a component of the strain tensor e. The coefficients $a_{\imath,}$ are obtained from static stress experiments (for example by Feher 1964).

In many cases the term quadratic in the strain is negligible. It will be neglected here, with the result that it will not be possible to predict the shape for transitions for which the linear term vanishes. The $\Delta M=2$ and two-phonon transitions for $\mathrm{MgO}: \mathrm{Fe}^{2+}$ fall into this category (McMahon 1964). We calculate the probability that the scalar

$$
\epsilon=\sum_{i j} a_{i j} e_{i j}
$$

lies between $\epsilon$ and $\epsilon+d \epsilon . \epsilon$ is the particular linear combination of strain components appropriate to the problem considered. We do not assume that the components of e are in some sense independent so that we may calculate the distributions of each component separately before performing a convolution appropriate to the problem. Although McMahon (1964) and Feher (1964) have shown that this is a useful working assumption, it is shown in the next section that the assumption is usually incorrect.

The position and any internal variables of the $i$ th source of strain will be denoted by $z_{2}$. For simplicity we assume that only one type of source is present; this will be generalized in $\S 3$ to cases where several types of strain source occur. If there are $N$ sources then

$$
\epsilon=\epsilon\left(z_{1}, z_{2}, \ldots, z_{N}\right)
$$


We further assume that the contributions of the various sources to $\epsilon$ add linearly:

$$
\epsilon=\sum_{i=1}^{N} \epsilon\left(z_{\imath}\right) \text {. }
$$

This assumption is fundamental. It should be valid when linear elasticity theory is valid, so that (4) should hold for strains less than about $10^{-2}$. The widths of observed strain-broadened lines correspond to strains of the order of $10^{-4}$, roughly two orders of magnitude less.

The variables $z_{1}, \ldots, z_{N}$ define a phase space. Each point in this phase space corresponds to a specific configuration of strain sources in which the position and the other parameters which describe every one of the sources have definite values. The statistical assumptions about the probability distribution of each of the $z_{l}$ are contained in a statistical weight function $p(z)$. The form of $p(z)$ depends on the species of source considered, but the functional form of $p\left(z_{i}\right)$ does not depend on $i$. A normalization factor $v$ is defined for $p(z)$ by

$$
v=\int d z_{\imath} p\left(z_{2}\right)
$$

We calculate the fraction of all configurations of the strain sources, consistent with our statistical assumptions, which produce a given value of $\epsilon$ at the point of observation. This is

$$
I(\epsilon)=\left(\frac{1}{v}\right)^{N} \int \ldots \int d z_{1} p\left(z_{1}\right) \ldots d z_{N} p\left(z_{N}\right) \delta\left\{\epsilon-\epsilon\left(z_{1}, \ldots, z_{N}\right)\right\}
$$

The delta function singles out the regions of the phase space for which $\epsilon\left(z_{1}, \ldots, z_{N}\right)$ has the value $\epsilon$. In particular this excludes configurations which produce infinite strains at the point of observation. For this reason singularities in $\epsilon(z)$ do not cause difficulty; such singularities are, however, very important in calculating the moments of $I(\epsilon)$, which are discussed in $\$ 3.3$.

$I(\varepsilon)$ can be calculated directly from (6) with no further physical assumptions. The actual procedure constitutes Markoff's method. Equation (6) may be rewritten using the spectral representation of the delta function:

giving

$$
\delta(y)=\frac{1}{2 \pi} \int_{-\infty}^{\infty} d x \exp (i x y)
$$

$$
I(\epsilon)=\left(\frac{1}{v}\right)^{N} \frac{1}{2 \pi} \int_{-\infty}^{\infty} d z \int \ldots \int d z_{1} p\left(z_{1}\right) \ldots d z_{N} p\left(z_{N}\right) \exp \left[i x\left\{\epsilon-\epsilon\left(z_{1}, \ldots, z_{N}\right)\right\}\right]
$$

where the spectral variable $x$ has no particular physical significance. From (4) the contributions of the sources to $\epsilon$ add linearly, so that (7) factorizes into

$$
I(\epsilon)=\frac{1}{2 \pi} \int_{-\infty}^{\infty} d x \exp (i x \epsilon)\left[\frac{1}{v} \int d z p(z) \exp \{-i x \in(z)\}\right]^{N} .
$$

Following Markoff (1912) and Margenau (1935) we write

where

$$
\frac{1}{v} \int d z p(z) \exp \{-i x \in(z)\}=1-\frac{1}{v} J(x)
$$

$$
J(x)=\int d z p(z)[1-\exp \{-i x \epsilon(z)\}]
$$


We finally obtain, recalling $(1-A / N)^{N} \rightarrow \exp (-A)$ in the limit of large $N$ :

$$
I(\epsilon)=\frac{1}{2 \pi} \int_{-\infty}^{\infty} d x \exp (i x \epsilon) \exp \{-\rho J(x)\}
$$

in which $\rho$, the density of the sources, is defined as

$$
\rho=\frac{N}{v}=\frac{N}{\int d z p(z)}
$$

in the limit of large $N$ and $v$. Equations (9), (10) and (11) give the distribution in magnitude of the microscope strain in terms of the strain field of each individual source $\epsilon(z)$, the statistical distribution of the sources $p(z)$ and $\rho$, the density of the sources.

The line shape described by (10) may be asymmetric. We conclude this section with the formulae equivalent to (9) and (10) in the especially useful case that the line is symmetrical about $\epsilon=0$ :

$$
\begin{aligned}
& J(x)=\int d z p(z)[1-\cos \{x \epsilon(z)\}] \\
& I(\epsilon)=\frac{1}{\pi} \int_{0}^{\infty} d x \cos (x \epsilon) \exp \{-\rho J(x)\} .
\end{aligned}
$$

\section{Discussion of the strain distribution}

In this section we derive a number of results based on (10). Firstly, we generalize (10) to the case where there are several distinct types of strain source. Secondly, we find the distribution of a sum of strain components $\epsilon=\epsilon_{1}+\epsilon_{2}$, and show that this is not the convolution of the distributions of $\epsilon_{1}$ and $\epsilon_{2}$ separately except in very special cases. The result obtained here can be used to calculate spin resonance line shapes which are broadened by both strain and by spin-spin interactions. Thirdly, the moments of $I(\epsilon)$ are calculated; these emphasize the advantages of calculating $I(\epsilon)$ directly rather than its moments. Finally, the line shape is shown to agree with the predictions of the central limit theorem in the special circumstances in which this theorem can be applied.

\subsection{Several types of strain source}

In real crystals the microscopic strains may be caused by several different species of strain source-for example both point defects and dislocations are important in radiationdamaged crystals. We shall assume that the statistical distributions of the various species are independent--thus the sources of species $\mathrm{A}$ are not correlated in position with those of type B. The discussion of $\S 2$ may be repeated with only minor changes. In particular the factorization of $(7)$ to give $(8)$ proceeds as before. $I(\epsilon)$ is again given by (10), but $\rho J(x)$ is now given by

$$
\rho J(x)=\rho_{\mathrm{A}} \int d z p_{\mathrm{A}}(z)\left[1-\cos \left\{x \epsilon_{\mathrm{A}}(z)\right\}\right]+\rho_{\mathrm{B}} \int d z p_{\mathrm{B}}(z)\left[1-\cos \left\{x \epsilon_{\mathrm{B}}(z)\right\}\right]
$$

where $\rho_{\mathrm{A}}=N_{\mathrm{A}} / \int d z p_{\mathrm{A}}(z)$ and $\rho_{\mathrm{B}}=N_{\mathrm{B}} / \int d z p_{\mathrm{B}}(z)$. In general $p_{\mathrm{A}}(z)$ and $p_{\mathrm{B}}(z)$ have different functional forms. 


\subsection{The distribution of a sum of strain components}

The detailed analyses of experimental line shapes by Feher and McMahon both assumed that the distributions of the various strain components are independent. If $\epsilon_{12}=\epsilon_{1}+\epsilon_{2}$, where the distribution of $\epsilon_{j}$ is given by $I_{j}\left(\epsilon_{j}\right)$, this assumption implies

$$
I_{12}(\epsilon)=\int_{-\infty}^{\infty} d s I_{1}(s) I_{2}(\epsilon-s) .
$$

It is straightforward to show from (10) that (12) is only valid if

in which

$$
J_{12}(x)=J_{1}(x)+J_{2}(x)
$$

$$
J_{2}(x)=\int d z p(z)\left[1-\exp \left\{-i x \epsilon_{2}(z)\right\}\right]
$$

In general (13) is satisfied only if the strain components $\epsilon_{1}$ and $\epsilon_{2}$ are produced by different species of strain source. This result can be seen more intuitively as follows. A large value of $\epsilon_{1}$ implies that the point of observation is close to one of the strain sources. If the source also produces the strain component $\epsilon_{2}$ this suggests that $\epsilon_{2}$ will also be large, so that the values of $\epsilon_{1}$ and $\epsilon_{2}$ will be correlated. As a more concrete example, screw dislocations produce no dilation in an isotropic crystal; in such a crystal containing only these strain sources $e_{x x}+e_{y y}+e_{z z}=0$, and it would clearly be incorrect to assume that $e_{x x}, e_{y y}$ and $e_{z z}$ were independent.

Equations (12) and (13) do hold in one case of practical importance. If a spin resonance line is appreciably broadened by both microscopic strains and by spin-spin interactions, as for $\mathrm{Co}^{2+}, \mathrm{Mn}^{2+}$ and $\mathrm{Fe}^{3+}$ in $\mathrm{MgO}$, the transition energy of one of these spins has the form

$$
\begin{aligned}
\hbar \omega & =\hbar \omega_{0}+\hbar \omega_{1} \epsilon+\hbar \omega_{\mathrm{s}} \epsilon_{\mathrm{s}} \\
& =\hbar \omega_{0}+\hbar \omega_{1}\left(\epsilon+\frac{\omega_{\mathrm{s}}}{\omega_{1}} \epsilon_{\mathrm{s}}\right)
\end{aligned}
$$

where the shift $\hbar \omega_{\mathrm{s}} \epsilon_{\mathrm{s}}$ is caused by spin-spin interactions. Grant and Strandberg (1964) have discussed the case in which there is spin-spin interaction broadening alone in some detail. If the magnetic defects produce no strain field then $\epsilon_{1} \equiv \epsilon$ and $\epsilon_{2} \equiv \omega_{\mathrm{s}} \epsilon_{\mathrm{s}} / \omega_{1}$ are produced by different species of imperfection and equations (12) and (13) hold. In this particularly simple case the resultant line shape is the convolution of the shape due to dipolar broadening alone with that due to strain broadening alone.

\subsection{The moments of $I(\epsilon)$}

In $\S 2$ the distribution of strains was calculated directly. It is common in the theory of line shapes to calculate the moments of a distribution instead. The moments of $I(\epsilon)$ are

$$
M_{n}=\frac{\int_{-\infty}^{\infty} d \epsilon I(\epsilon) \epsilon^{n}}{\int_{-\infty}^{\infty} d \epsilon I(\epsilon)}
$$

These are not measured experimentally, their one advantage being that in some cases they may be calculated without difficulty. For strain-broadened lines even this advantage 
is lacking, for the moments are dominated by the regions of large $\epsilon$ and are not even finite unless great care is taken. The moments can be expressed in terms of integrals $S_{n}$, where

$$
S_{n}=\rho \int d z p(z)\{\epsilon(z)\}^{n}
$$

by use of a general result of Kubo and Tomita (1954) which gives, in the present case,

The first four moments are

$$
M_{n}=\lim _{x \rightarrow 0} \frac{(-1)^{n} d^{n}}{d(i x)^{n}} \exp \{-\rho J(x)\}
$$

$$
\begin{aligned}
& M_{1}=S_{1} \\
& M_{2}=S_{2}+S_{1}^{2} \\
& M_{3}=S_{3}+3 S_{1} S_{2}+S_{2}^{3} \\
& M_{4}=S_{4}+4 S_{1} S_{3}-2 S_{1}{ }^{2}+3\left(S_{2}+S_{1}^{2}\right)^{2} .
\end{aligned}
$$

For symmetric lines it is easily shown from (15) that $S_{n}$ is zero if $n$ is odd. The odd moments vanish and the even moments simplify to give, for example,

$$
\begin{aligned}
& M_{2}=S_{2} \\
& M_{4}=3 S_{2}^{2}+S_{4} .
\end{aligned}
$$

For a Gaussian line shape $M_{4}=3 M_{2}^{2}$, which would require $S_{4}$ to be zero.

\subsection{Relation to the central limit theorem}

It is sometimes believed that a strain-broadened line shape should be Gaussian, a belief which ultimately stems from the central limit theorem which is fundamental in the theory of errors (Kendall and Stuart 1958, p. 193). In this subsection we show that $I(\epsilon)$ given by $(9)$ and $(10)$ indeed gives a Gaussian shape in the circumstances in which the theorem is applied.

The theorem is usually based on two assumptions. Firstly, the contributions of each of the sources should be equal in magnitude, having positive and negative signs with equal probability:

$$
\epsilon\left(z_{i}\right)= \pm \varepsilon_{0} \text {. }
$$

Secondly, the local strain at the point of observation must be produced by a large number of sources. Thus $\epsilon_{0}$ will be much smaller than the half-width of the distribution $\epsilon_{\mathrm{w}}$. Although results similar to the central limit theorem hold in more general circumstances than these (Chandrasekhar 1943) we shall only consider the case commonly considered in the theory of errors.

For a Gaussian shape we see from (10) that $\rho J(x)$ must be proportional to $x^{2}$. The first condition, (16), shows that the line is symmetrical and, by $\left(9^{\prime}\right)$ and (11),

$$
J(x)=N\left\{1-\cos \left(x \epsilon_{0}\right)\right\} \text {. }
$$

The values of $x$ which are of dominant importance in (10) are of order $1 / \epsilon_{\mathrm{w}}$, for which $x \epsilon_{0} \sim \epsilon_{0} / \epsilon_{\mathrm{W}}$ is small by the second assumption. Thus

$$
J(x)=\frac{1}{2} N \epsilon_{0}{ }^{2} x^{2}
$$

confirming the Gaussian shape under the rather restrictive conditions assumed. 


\section{A statistically isotropic and homogeneous distribution of dislocations}

As a specific example we consider an elastically isotropic crystal in which the only strain sources are straight edge or screw dislocations. Each of the dislocations is described by five variables $r, b, \theta, \phi$ and $\alpha$ which correspond to the $z_{\imath}$ used earlier. These variables are shown in figure $1 . r$ is the distance from the point of observation to the

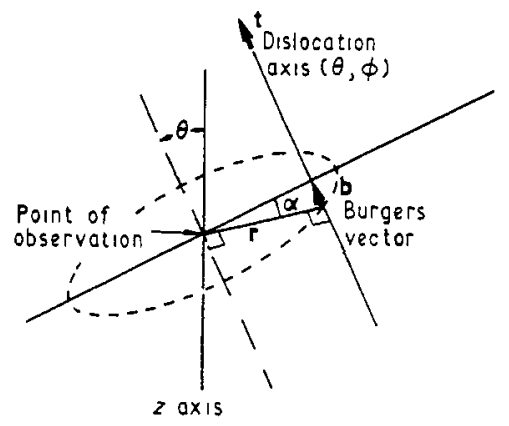

Figure 1. The notation of $\S 4$ for a screw dislocation.

nearest point of the dislocation line, $b$ the magnitude of the Burgers vector, and the dislocation axis $\mathbf{t}$ is specified by the angles $\theta$ and $\phi$. For edge dislocation $\alpha$ is the angle between $\mathbf{b}$ and $\mathbf{r}$, whereas for screw dislocations $\alpha$ is shown in figure 1 .

We assume that the distribution of the defects whose transitions are observed is completely uncorrelated with the dislocation distribution. The statistical distribution of dislocations will be assumed to be isotropic and homogeneous. By this we mean that both the axes and Burgers vectors of the dislocations are distributed isotropically and that, for dislocations of given $b$ and $t$, the points of intersection of the dislocations with an arbitrary plane are distributed homogeneously on the plane. For an isotropic distribution the probability that the dislocation axis lies within a particular solid angle is simply proportional to the solid angle, i.e. to $d \theta \sin \theta d \phi$. For a distribution which is homogeneous the probability of given values of $r$ and $\alpha$ is proportional to $d r \gamma d \alpha$. Thus integrals of the form $\int d z p(z) f(z)$, where $f$ is an arbitrary function of $z$, should here be interpreted as

$$
\int_{0}^{\pi} d \theta \sin \theta \int_{0}^{2 \pi} d \phi \int_{0}^{2 \pi} d \alpha \int_{R_{1}}^{R_{2}} d r r f(\theta, \phi, \alpha, r) .
$$

A constant of proportionality has been omitted, but this simply cancels in calculating the line shape.

The lower limit $R_{1}$ on the radial integral takes account of the atomic structure of the lattice. In calculating $I(\epsilon)$ it is a good approximation to put $R_{1}=0$; this would not be possible if the moments of $I(\epsilon)$ were needed as the strain field of the dislocation diverges as $1 / r$ as $r$ becomes small. The upper limit $R_{2}$ simply means that we consider a finite crystal; as $R_{2}$ becomes infinite the usual logarithmic divergences met in dislocation theory occur. As a finite crystal is used the distribution $I(\epsilon)$ will depend on the geometry of the surface for the very small values of $\epsilon$ for which the dislocations near the surface are important. Thus $I(\epsilon)$ should be unreliable when $\epsilon$ is less than about $b / R_{2}$, or about $10^{-6}$ or $10^{-7}$. Recalling that (4) is only valid for strains $\epsilon$ less than about $10^{-2}$, as linear theory of elasticity has been assumed, we see that the calculated distribution of strains 
$I(\epsilon)$ will only be reliable for

$$
10^{-2} \gtrsim \epsilon \geq 10^{-6} \text {. }
$$

As the observed half-widths of $I(\epsilon)$ are of order $10^{-4}$ the inequality (18) does not provide any real restriction in practice.

We now outline the calculation of $\rho, J(x)$ and $I(\epsilon)$. Throughout we will assume $R_{1}$ to be zero. The value of (17) for a sphere of radius $R$ and $f=1$ is

$$
\int d z p(z)=4 \pi^{2} R^{2}
$$

The total length of dislocation per unit volume will be denoted $L$. The total length of dislocation within the sphere is thus $\frac{4}{3} \pi R^{3} L$. It is easily shown that for an isotropic homogeneous distribution the mean of the lengths of each dislocation within the crystal is $\frac{4}{3} R$, so that $N=\pi R^{2} L$ dislocations cut the sphere. Thus

$$
\rho=\frac{N}{\int d z p(z)}=\frac{L}{4 \pi}
$$

which is, of course, independent of $R$.

The strain field of a straight dislocation in an elastically isotropic crystal can be written

$$
\epsilon(z)=\frac{b}{\pi r} \psi(\theta, \phi, \alpha)
$$

where $\psi$ depends on the particular combination of strain components required and on the edge or screw nature of the dislocation. $J(x)$ is given by

$$
J(x)=\int_{0}^{\pi} d \theta \sin \theta \int_{0}^{2 \pi} d \phi \int_{0}^{2 \pi} d \alpha \int_{R_{1}}^{R_{2}} d r r\left\{1-\cos \left(x \frac{b}{\pi r} \psi\right)\right\} .
$$

It is shown in the appendix that, if we again assume $R_{1}$ zero, (19) and (20) together lead to

$$
\rho J(x)=x^{2} L b^{2}(A-B \ln |x|) .
$$

The line shape is given by $\left(10^{\prime}\right)$ and $(22)$ as

$$
I(\epsilon)=\frac{1}{\pi} \int_{0}^{\infty} d x \cos (\epsilon x) \exp \left\{-x^{2} L b^{2}(A-\bar{B} \ln x)\right\} .
$$

If $B$ were zero this would describe a Gaussian shape, for which $\rho J(x)$ is proportional to $x^{2}$; the width of this Gaussian would be proportional to the square root of the dislocation density $L$. The term $B \ln (x)$ has the effect of reducing $I(\epsilon)$ at larger values of $\epsilon$.

$I(\epsilon)$ is determined qualitatively by the innermost (radial) integral in $J(x)$, and not by the subsequent angular integrals over $\theta, \phi$ and $\alpha$. Although the magnitudes of $A$ and $B$ depend on the edge or screw nature of the dislocations considered and on the particular sum of strain components constituting $\epsilon$, the functional forms of (22) and (23) do not depend on these factors. Thus if an observed line shape, apparently caused by dislocations alone, has a shape qualitatively different from (23), either the dislocation distribution is inhomogeneous or the centres observed (which sample the strain field) are correlated in position with the dislocations. In practice dislocations are often concentrated in subgrain boundaries, giving a strongly inhomogeneous distribution. Further, point defects, analogous to the centres observed, are quite often found to be bound to 
dislocations; examples of this are given in the review by Newman and Bullough (1963). If the centres are magnetic ions of the correct charge and crystal radius (e.g. $\mathrm{Co}^{2+}$ in $\mathrm{MgO}$ ) then they will presumably be distributed very nearly at random, and the inhomogeneity of the dislocation distribution will be more important. On the other hand, colour centres produced by irradiation, especially vacancies with trapped electrons and holes, may have zero phonon lines broadened largely by the strain fields of other point defects produced at the same time. Some correlation between the positions of the centres observed and the strain sources would be expected in this case. If the correlation is important both the line shape and its width should differ from values predicted neglecting correlation.

The assumption of isotropy can also be checked, although less directly. $I(\epsilon)$ can sometimes be measured for two different sums of strain components, $\epsilon_{1}$ and $\epsilon_{2}$. The relative widths of $I_{1}\left(\epsilon_{1}\right)$ and $I_{2}\left(\epsilon_{2}\right)$ depend on the relative values of $A$ and $B$ in the two cases; these in turn are sensitive to the extent to which the distribution of dislocations is anisotropic.

\section{The $\Delta M=1$ line of $\mathrm{MgO}: \mathrm{Fe}^{2+}$}

The broad $\Delta M=1$ line of the spin resonance spectrum of $\mathrm{Fe}^{2+}$ in $\mathrm{MgO}$ has been shown by McMahon to be broadened by the microscopic strain mechanism under discussion. The line is several hundreds of gauss wide, varying with the magnetic field orientation (Lewis 1965) and roughly Lorentzian in shape (McMahon 1964). A near Lorentzian shape is also found for the ENDOR line of $\mathrm{Co}^{2+}$ in $\mathrm{MgO}$ (Fry and Llewellyn 1962) which is again almost entirely strain broadened. The shape is thus different from that expected from (23). This difference can be attributed to the subgrain structure of the distribution of dislocations in $\mathrm{MgO}$ (Lang and Miuscov 1964), a feature which was entirely neglected in the simple distribution assumed in deriving (23). The line shape due to a distribution with subgrain structure is to be treated in a later publication; here we will continue to discuss the isotropic homogeneous distribution.

The analogue of (1) for $\mathrm{MgO}: \mathrm{Fe}^{2+}$ with the magnetic field along the (001) axis is

$$
\hbar \omega=\hbar \omega_{0}+\frac{3}{4} G_{11} \epsilon_{001}
$$

where $\epsilon_{001}=2 e_{z z}-e_{x x}-e_{y y}$; with the field in the (111) direction the corresponding result is

$$
\hbar \omega=\hbar \omega_{0}+2 G_{44} \epsilon_{111}
$$

in which $\epsilon_{111}=e_{x y}+e_{y z}+e_{z x}$. We do not adopt the engineering convention for strains. $G_{11}$ and $G_{44}$ are coupling coefficients (Shiren 1962, Watkins and Feher 1962).

The elastic constants of the crystal are altered near to the impurities whose transition energies measure the strain field. The strains we have been discussing are, however, those which would occur if the local elastic constants were unaltered. This is an apparent, rather than a real, difficulty, for transition energies and not strains are measured directly. The $G_{i j}$ used in (24) and (25) are found from static stress experiments, using the perfect crystal elastic constants to convert stresses to strains. To use the $G_{i j}$ consistently to calculate changes in transition energies we must therefore make no allowance for changes in the local elastic properties when discussing microscopic strain broadening. It is convenient to quote all results in terms of strains for purposes of comparison; in all cases these will be reduced to values appropriate to a lattice with the elastic moduli of a perfect crystal. To convert these strains into actual atomic positions near the defect the altered local force constants must, of course, be included. 
The full-widths at half intensity of the distributions of $\epsilon_{001}$ and $\epsilon_{111}$ can be found from an analysis of the various spin resonance line shapes. These are shown in the table; in all cases the analysis is that of the author. For $\mathrm{Co}^{2+}$ the coupling coefficients of Tucker (1966) were used. There are, unfortunately, large errors in the coupling coefficients $G_{11}$ and $G_{44}$ and in estimating the strain contribution to the observed linewidth. The $\epsilon_{001}$ width is about $2 \times 10^{-4}$ and that of $\epsilon_{111}$ about $0.6 \times 10^{-4}$. Clearly there will be differences from specimen to specimen, depending on the method of formation and on the mechanical history of the crystal used.

\section{The magnitudes of the full-widths at half intensity of $I(\epsilon) \dagger$}

$\begin{array}{lcccc}\text { Experiment } & \text { Reference } & \epsilon_{001} & \epsilon_{111} & \epsilon_{001} / \epsilon_{111} \\ \mathrm{Fe}^{2+} \text { ESR } & \mathrm{a} & 20 & 060 & 3 \cdot 2 \\ & \mathrm{~b} & 17 & 069 & 25 \\ \mathrm{Fe}^{3+} \text { ESR } & \mathrm{c} & 21 & 053 & 40 \\ \mathrm{Mn}^{2+} \text { ESR } & \mathrm{c} & 2 \cdot 0 & 064 & 3 \cdot 1 \\ \mathrm{Co}^{2 \div} \text { ESR } & \mathrm{b} & 18 & & \\ \mathrm{Co}^{2 \div} \text { ENDOR } & \mathrm{d} & 23 & & \\ \text { Theory } & \mathrm{e} & 28 & 28 & 1 \cdot 0 \\ & \mathrm{f} & 0.7 & 03 & 2 \cdot 3\end{array}$

The strains are in units of $10^{-4}$.

The experimental sources are: $a$, Lewis $1965 ; \mathrm{b}$, McMahon 1964; c, Feher 1964; d, Whittlestone 1964, Ph.D. Thesis, Unversity of Bristol; e, Fry and Llewellyn 1962; f, present paper.

$E S R$, electron spin resonance.

+ Calculated from a variety of spin resonance data for ions in $\mathrm{MgO}$ for

$$
\begin{aligned}
& \epsilon_{001}=2 e_{z z}-e_{z x}-e_{y y} \\
& \epsilon_{111}=e_{x y}+e_{y z}+e_{z z}
\end{aligned}
$$

in which we do not use engineering notation.

In calculating the strain distributions we assume that the Burgers vectors are equally divided among the (110) and equivalent directions, and that the dislocation axes are isotropically distributed. This is a simple generalization of $\$ 4$, where the Burgers vectors were also isotropic. Thus the strain field of a dislocation whose axis makes an angle $\eta$ with its Burgers vector is expressed in terms of the strain fields of screw and edge dislocations with the same Burgers vector and corresponding axes as

$$
e_{i j}=e_{2 j}{ }^{\text {(screw) }} \cos \eta+e_{2 j}{ }^{\text {(edge) }} \sin \eta \text {. }
$$

The calculated distributions are shown in figure 2 for a dislocation density of $5 \times 10^{5} \mathrm{~cm}$ per $\mathrm{cm}^{3}$. The $\epsilon_{001}$ and $\epsilon_{111}$ widths are $0.7 \times 10^{-4}$ and $0.3 \times 10^{-4}$ respectively, which would correspond to linewidths at $X$ band of 250 and 175 gauss. Although these are of the right order of magnitude they are rather less than the observed widths of about 600 and 350 gauss. To give the same half-widths a dislocation density $L$ of about $3 \times 10^{6} \mathrm{~cm}$ per $\mathrm{cm}^{3}$ would be required, as the linewidth varies roughly as $\sqrt{ } L$. This is about an order of magnitude larger than that observed by Lang and Miuscov. Certainly a part (and probably most) of the discrepancy can be attributed to the neglect of the subgrain structure, which is also responsible for the differences of the observed and calculated line shapes, mentioned earlier. It should also be recalled, however, that the measured densities were for crystals of high perfection, and may not be typical of the 
crystals used here. Further, the observed densities are particularly high in the subgrain boundaries, so that limits of experimental resolution may have led to low estimates.

Despite the differences between the observed and calculated widths, the ratio $\epsilon_{001} / \epsilon_{111}$ is about $2 \cdot 3$, and is within the rather broad limits set by experiment. Thus the assumption of (110) Burgers' vectors and isotropic distribution of axes of dislocation seems to be verified.

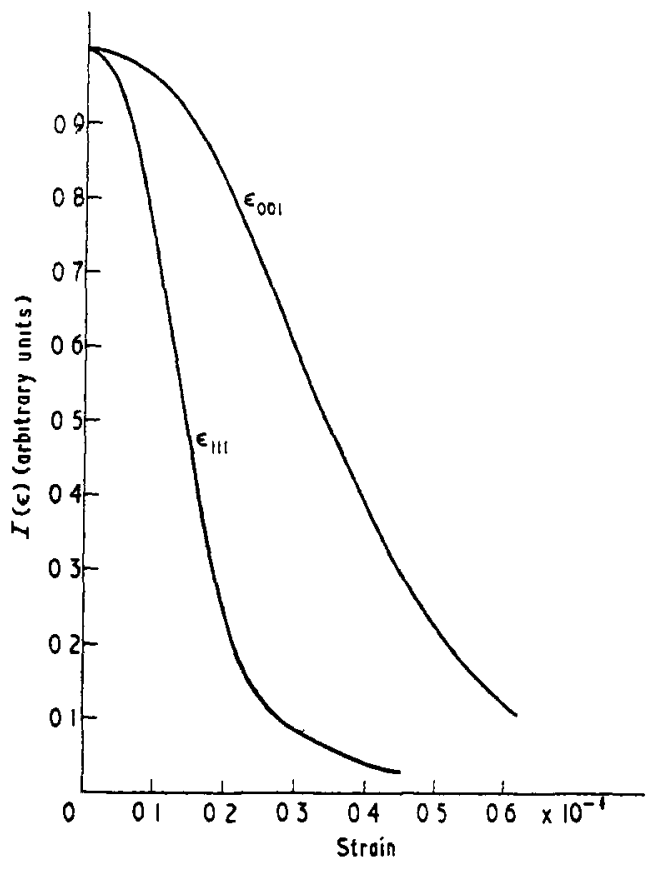

Figure 2. The form of $I(\epsilon)$ in two cases, viz.

$$
\epsilon_{001}=2 e_{z z}-e_{x x}-e_{y y} \text { and } \epsilon_{111}=e_{z y}+e_{y z}+e_{z x} .
$$

These curves assume for the dislocations a homogeneous distribution in space with $L=5 \times 10^{5} \mathrm{~cm}$ per $\mathrm{cm}^{3}$. The dislocation axes are 1sotropically distributed and the Burgers vectors lie in the $\langle 110\rangle$ and equivalent directions.

\section{Conclusion}

A general method for calculating strain-broadened line shapes has been outlined. The line shape is expressed in terms of the density and statistical distribution of the sources of strain, and in terms of the strain fields of the individual strain sources. The method is capable of direct extension to cases where the line broadening has other contributions, such as those from spin-spin interactions. The present work does not assume any arbitrary form for the distribution of the microscopic strains, nor does it assume that the components of the microscopic strain tensor are statistically independent; previous workers make both these assumptions (Feher 1964, McMahon 1964).

To illustrate the method the line shape and linewidth for the electron spin resonance of $\mathrm{Fe}^{2+}$ in $\mathrm{MgO}$ was calculated, assuming an isotropic homogeneous distribution of dislocations. Although the results are generally in fair agreement with experiment the neglect of the subgrain structure in $\mathrm{MgO}$ affects both the width and the shape noticeably. 
Calculations taking account of the subgrain structure are in progress. This sensitivity to the dislocation structure is in fact a real advantage, for it suggests that the observation of spin resonance and zero phonon line shapes can give useful information on the statistical distribution of dislocations and other imperfections in crystals.

\section{Acknowledgments}

The author wishes to thank Dr. A. B. Lidiard, Dr. R. Bullough, Dr. B. Henderson, Mr. A. E. Hughes and Mr. I. K. Ludlow for helpful discussions and for the use of unpublished data.

\section{Appendix. The evaluation of $J(x)$}

$J(x)$ is given by (21). The innermost integral over $r$ may be rewritten in terms of $v=r / R_{2}$ and $U=x\left(b / \pi R_{2}\right) \psi$ as

where

$$
R_{2}^{2} \int_{R_{1} / R_{2}}^{1} d v v\left(1-\cos \frac{U}{v}\right)=\frac{1}{2} R_{2}^{2} U^{2}\left\{f(U)-f\left(\frac{U R_{2}}{R_{1}}\right)\right\}
$$

$$
f(t)=\frac{1-\cos t}{t^{2}}+\frac{\sin t}{t}-\operatorname{Ci}(t)
$$

It is easy to show that it is a good approximation to take $R_{1}=0$. In this case (A1) may be simplified using the asymptotic form of the cosine integral $\mathrm{Ci}(t)$ (Jahnke and Emde 1945) for small $t$. (A1) becomes

$$
\frac{1}{2} R_{2}^{2} U^{2}\left(0.9228+\ln \frac{1}{|U|}\right)
$$

Combining (19), (21) and (A2)

$$
J(x)=x^{2} L b^{2}(A-B \ln |x|)
$$

where

$$
\begin{aligned}
& A=\left[\left\{0.9228+\ln \left(\frac{\pi R_{2}}{b}\right)\right\} I_{2}+I_{3}\right] \frac{1}{8 \pi^{3}} \\
& B=\frac{I_{2}}{8 \pi^{3}} .
\end{aligned}
$$

The angular integrals $I_{2}$ and $I_{3}$ are

and

$$
I_{2}=\int_{0}^{\pi} d \theta \sin \theta \int_{0}^{2 \pi} d \phi \int_{0}^{2 \pi} d \alpha|\psi(\theta, \phi, \alpha)|^{2}
$$

$$
I_{3}=-\int_{0}^{\pi} d \theta \sin \theta \int_{0}^{2 \pi} d \phi \int_{0}^{2 \pi} d \alpha|\psi|^{2} \ln |\psi| .
$$

The integrals $I_{2}$ and $I_{3}$ were evaluated on the Atomic Energy Authority STRETCH computer system. 


\section{References}

ANderson, P. W., 1951, Phys. Rev., 82, 342.

ChaNdrasekhar, S., 1943, Rev. Mod. Phys., 15, 1-89.

Feger, E. R., 1964, Phys. Rev., 136, A145-57.

FRY, D. J. I., and Llewellyn, P. M., 1962, Proc. Roy. Soc, A, 226, 84-94.

Grant, W. J. C., and Strandberg, M. W. P., Phys. Rev., 135, A715-26.

Hughes, A. E., and Runciman, W. A., 1965, Proc. Phys. Soc., 86, 615-27.

JAFNTKE, E., and ENDE, F., 1945, Tables of Functions (New York: Dover Publications).

Kendall, M. F., and Stuart, A., 1958, Advanced Theory of Siatistics, Vol. I (London: Griffin).

Kubo, R., and Tomita, K., 1954, J. Phys. Soc. Japan, 9, 888-919.

Lang, A. R., and Mruscov, V. F., 1964, Phil. Mag., 10, 263-8.

LEWIS, M. F., 1965, Phys. Letters, 19, 459-60.

Ludlow, I. K., and Runcrian, W. A., 1965, Proc. Phys. Soc., 86, 1081-6.

McMahon, D. H., 1964, Phys. Rev., 134, A128-39.

Margenat, H., 1935, Phys. Rev., 48, 755-65.

MARKOFF, A. A., 1912, Wahrscheinlichkeitsrechnung (Leipzig: Teubner).

Newman, R. C., and Bullough, R., 1963, Progr. in Semicond., 7, 101-34.

SHIREN, N. S., 1962, Bull. Amer. Phys. Soc., 7, 29.

TuckER, E. B., 1966, Phys. Rev., 143, 264-74.

Watkins, G., and FeHER, E., 1962, Bull. Amer. Phys. Soc., 7, 29. 